\title{
Medullar Infarcts of the Posterior Cervical Spinal Area: About an Observation in the Neurology Department of the University Hospital of Cocody, Abidjan, Ivory Coast
}

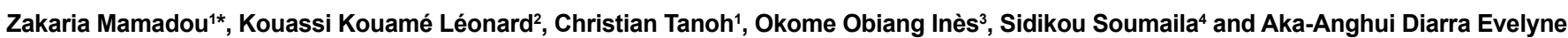

${ }^{1}$ Department of Neurology, University Hospital of Cocody, Abidjan, Ivory Coast

${ }^{2}$ Department of Neurology, University Hospital of Yopougon, Abidjan, Ivory Coast

${ }^{3}$ Department of Physical Medicine and Re-Adaptation, University Hospital of Yopougon, Ivory Coast

${ }^{4}$ Department of Medical Imagery, University Hospital of Treicheville, Abidjan, Ivory Coast

\begin{abstract}
Medullar infarcts are rare and are responsible for various neurological deficits that may consist of severe paraplegia or quadriplegia. The incidence of medullar infarcts is very low compared to that of cerebral infarcts.

We report a case of medullar infarct of the posterior spinal cervical area observed in a 55-year-old patient hospitalized in the neurology department of the University Hospital of Cocody, Abidjan, Ivory Coast.
\end{abstract}

Keywords: Medullar infracts; Posterior cervical spinal area; Ivory Coast

\section{Introduction}

Medullar infarcts are rare and are responsible for various neurological deficits that may consist of severe paraplegia or quadriplegia.

The incidence of medullar infarction is very low compared to that of cerebral infarcts [1]. Its prevalence is estimated to $6 \%$ of all acute myelopathies and only $1 \%$ to $2 \%$ of neurovascular pathologies [2]. The magnetic resonance imaging is the best exam to exclude other causes of acute spinal cord syndromes and to visualize infarct. The causes of medullar ischemia are as numerous as those of cerebral ischemia, and it is common not to find any. The symptoms may be transient or permanent [3].

We report here a case of cervical medullar infarct observed in a patient hospitalized in the neurology department of the University Hospital of Cocody, Abidjan, Ivory Coast.

\section{Case Report}

It is about a 55-year-old patient with right-sided lateral aneurysm with regular blood pressure, admitted to the neurology department of the University Hospital of Cocody for a motor deficit of the four members of brutal installation without any particular context. The

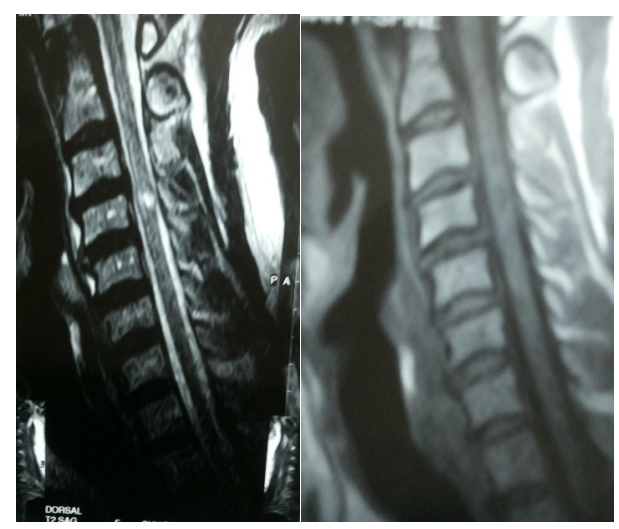

Figure 1: MRI cervical spine EST2 and EST1. clinical examination found a predominantly proximal flasco-spastic tetraparesis rated $2 / 5$ and $3 / 5$ distally according to the Medical Research Council (MRC) scale, an arthro-kinetic and vibratory anesthesia, an upper cervical hypoesthesia and a proprioceptive ataxia. The examination did not record any vesico-sphincteric disorder, no root syndrome or spinal syndrome. The whole clinical picture evoked a combined sclerosis of the marrow. The remainder of the clinical examination was unusual.

A medullar MRI (magnetic resonance imaging) was performed a week later. It was possible to demonstrate a hyper-signal sequence in EST2 and STIR, and a hypo-signal in EST1 not enhanced by the gadolinium of the posterior area of the cervical spinal cord compared to the fourth cervical vertebra (Figure 1). These clinical and radiological data evoked several mechanisms, inflammatory, infectious, vascular or metabolic.

In the context of infectious and inflammatory etiology, a microbiological analysis of the cerebrospinal fluid was performed and did not reveal either pleiecytosis or intra-techal synthesis. The CRP was also negative.

Ablood-plate count (BPC) and the B9, B12 vitamin assay for Biermer's disease were strictly normal. The negativity of the aforementioned examinations and the abruptness of the symptomatology were in favor of an ischemic vascular accident of the posterior cervical spinal area. The etiological assessment (Doppler ultrasound of the supra-aortic trunks, cardiac ultrasound, trans-esophageal doppler ultrasound and electrocardiogram) was normal. A secondary prevention treatment included symptomatic treatment and hygiene-dietetic measures. Thus,

*Corresponding author: Zakaria Mamadou, Department of Neurology, University Hospital of Cocody, Abidjan, Ivory Coast, Tel: 0022577325200; E-mail: mamadouzakaria1@hotmail.fr

Received April 23, 2017; Accepted May 09, 2017; Published May 11, 2017

Citation: Mamadou Z, Léonard KK, Tanoh C, Inès OO, Soumaila S, et al. (2017) Medullar Infarcts of the Posterior Cervical Spinal Area: About an Observation in the Neurology Department of the University Hospital of Cocody, Abidjan, Ivory Coast. J Neurol Disord 5: 341. doi:10.4172/2329-6895.1000341

Copyright: (c) 2017 Mamadou Z, et al. This is an open-access article distributed under the terms of the Creative Commons Attribution License, which permits unrestricted use, distribution, and reproduction in any medium, provided the original author and source are credited. 
Citation: Mamadou Z, Léonard KK, Tanoh C, Inès OO, Soumaila S, et al. (2017) Medullar Infarcts of the Posterior Cervical Spinal Area: About an Observation in the Neurology Department of the University Hospital of Cocody, Abidjan, Ivory Coast. J Neurol Disord 5: 341. doi:10.4172/23296895.1000341

Page 2 of 2

the patient was given an anti-aggregant platelet (acetylsalicylic acid 100 $\mathrm{mg} /$ day), an antihypertensive agent, pregabalin $150 \mathrm{mg}$ per OS and physical rehabilitation (physiotherapy sessions for muscle, joint and transfer work were undertaken and carried out for several months).

The evolution was marked after three (3) months by a partial motor recovery of the four limbs of $2 / 5$ to $4 / 5$ in proximal and $3 / 5$ to $5 / 5$ in distal according to the MRC scale. There was also a partial recovery of superficial and deep sensitivity.

\section{Discussion}

The extreme rarity of medullar infarct compared to cerebral infarct is explained by the small size of the marrow, the wealth of the arterial anastomoses and the low incidence of atheroma on the medullar arteries.

Medullar vascularization is one of the most complex in the body because of the large number of arteries composing it $[4,5]$. The medulla is vascularized by an anterior spinal artery, median, and two lateral posterior spinal arteries. There is medullar ischemia by the affection of the anterior spinal artery, but very rarely of the posterior spinal artery. The anterior spinal artery vascularizes the central area of the medullar cord. The posterior spinal arteries vascularize the periphery of the marrow and constitute a peri-medullar anastomotic pie-meri mantle. Due to the multiple afferents feeding the posterior spinal arteries, infarcts in this area are rare [6]. Our patient has an infarct in a posterior spinal artery. Very few cases had been described before the MRI [6-8].

The Clinical semiology is highly variable due to the complex anastomotic networks that are located in that area. [3] Most of these infarcts are bilateral [9]. They are then mainly responsible for sensory disorders of paresthesia type and abolition of vibratory sensitivity in the lesion area. However, paraparesis or tetraparesis by affection of the pyramidal bundles is possible. Our patient presents a symptomatology typical of the affection of the posterior spinal cervical areas, namely a tetraparesis with deep sensitivity disorder (vibratory and arthrokinetic) and superficial (thermo-algic).

The diagnosis of a medullar infarct should not be a diagnosis of elimination, but should be affirmed on the basis of an evocative clinical picture and an MRI.

The abrupt onset of neurological deficit requires an emergency MRI. The characteristics of medullar infarcts are similar to those of cerebral infarcts. In the acute phase, the medullar infarct can go unnoticed because the lesion is iso-intense in T1 sequences, and the T2 hyperactivity is detectable only from the $6^{\text {th }}$ hour [10]. A few days to a few weeks after the onset, the edema worsens and leads to the appearance of a hypo-signal in T1-weighted sequences, and a frank hyper-signal with an increase in the caliber of the marrow on the sagittal sections [11] and axial lesions centered on the lesion. These same neuro-radiological lesions (MRI) were observed in our patient with the exception of the increase in the size of the spinal cord.

Most medullar infarcts are the result of a condition affecting a large or medium-sized artery, usually the aorta. They are much more rarely due to an embolism or an intrinsic affection of the small intra-medullar arteries. Despite the progress of the methods of investigation, it is common not to identify a cause. This is the case in our patient where no cause has been identified.

Independently of the treatment of the cause, the treatment of the medullar infarcts is symptomatic: the rehabilitation is the base of the treatment. No treatment has been tested to date. The Heparin is usually recommended, if only to reduce the extent of venous thrombosis [12].

The prognosis of medullar infarcts remains severe. The acute mortality is about $20 \%$ and severe squeal are present in $50 \%$ to $60 \%$ of patients [13]. In contrast, the evolution was favorable in our patient with good motor recovery.

\section{Conclusion}

The cerebral infarction remains rare. The diagnosis is based on a cluster of clinical (abrupt symptomatology) and neuro-radiological (medullar MRI) which has revolutionized the diagnosis. The etiologies are multiple and the early care is at first symptomatic in order to improve the functional prognosis.

\section{References}

1. Pelser H, Van Gijn J (1993) Spinal infarction: A follow-up study. Stroke 24: 896898.

2. Sandson TA, Friedman JH (1989) Spinal cord infarction: A report of 8 cases and review of the literature. Medicine 68: 282-292

3. Hughes JT (1989) Vascular disorders of the spinal cord. Handbook of clinical neurology. Vascular Diseases. Part III. Elsevier Science Publisher, Amsterdam, The Netherlands.

4. Thron AK (1988) Vascular anatomy of the spinal cord. Neuroradiological investigations and clinical syndromes. Berlin: Springer-Verlag p: 65-105.

5. Lasjaunias P, Berenstein A (1991) Surgical neuro-angiography. Vol. 3. Functional vascular anatomy of brain, spinal cord and spine p: 337.

6. Perier O, Demanet JC, Henneaux J, Vincente NA (1960) Existe-t-il un syndrome des artères spinales postérieures? À propos de deux observations anatomocliniques. Rev Neurol 103: 396-409.

7. Chung MF (1926) Thrombosis of the spinal vessels in sudden syphilitic paraplegia. Arch Neurol Psychiatry 16: 761-771.

8. Gruner J, Lapresle J (1962) Étude anatomo-pathologique des médullopathies d'origine vasculaire. Rev Neurol 106: 592-631.

9. Bergqvist CA, Goldberg HI, Thorarensen O, Bird SJ (1997) Posterior cervical spinal cord infarction following vertebral artery dissection. Neurology 48: 11121115.

10. Sibon I, Menegon P, Moonen CT, Dousset V (2003) Early diagnosis of spina cord infarct using magnetic resonance diffusion imaging. Neurology 61: 1622.

11. Mawad ME, Rivera V, Crawford S, Ramirez A, Breitbach W (1990) Spinal cord ischemia after resection of thoracoabdominal aortic aneurysms: MR findings in 24 patients. AJNR Am J Neuroradiol 11: 987-991.

12. Leys D, Weerts J, Pruvo JP (1998) Spinal ischemic strokes. Cerebrovasc Dis 111: $1560-1568$

13. Masson C, Pruvo JP, Meder JF, Cordonnier C, Touzé E, et al. (2004) Spinal cord infarction: Clinical and MRI findings and short-term outcome. J Neurol Neurosurg Psychiatry 75: 1431-1435. 